CORRIGENDUM

\title{
Corrigendum: Neurexins 1-3 Each Have a Distinct Pattern of Expression in the Early Developing Human Cerebral Cortex
}

\author{
Lauren F. Harkin 1,2,3, Susan J. Lindsay², Yaobo Xu²,4, Ayman Alzu'bi'1,2, \\ Alexandra Ferrera ${ }^{1,2}$, Emily A. Gullon ${ }^{1,2}$, Owen G. James ${ }^{1,2,5}$ and Gavin J. Clowry ${ }^{1}$
}

${ }^{1}$ Institute of Neuroscience, Newcastle University, Framlington Place, Newcastle upon Tyne NE2 4HH, UK, ${ }^{2}$ Institute of Genetic Medicine, Newcastle University, International Centre for Life, Parkway Drive, Newcastle upon Tyne NE1 3BZ, UK, ${ }^{3}$ Present address: School of Healthcare Science, Manchester Metropolitan University, Manchester M1 5GD, UK, ${ }^{4}$ Present address: Wellcome Trust, Sanger Institute, Cambridge CB10 1SA, UK and ${ }^{5}$ Present address: MRC Centre for Regenerative Medicine, University of Edinburgh, Edinburgh EH16 4UU, UK

Address correspondence to Gavin J. Clowry, Institute of Neuroscience, Newcastle University, Framlington Place, Newcastle upon Tyne NE2 4HH, UK. Email: gavin.clowry@ncl.ac.uk or Susan J. Lindsay, Institute of Genetic Medicine, Newcastle University, International Centre for Life, Parkway Drive, Newcastle upon Tyne NE1 3BZ, UK. Email: susan.lindsay@ncl.ac.uk

Cerebral Cortex, Volume 27, Issue 1, 1 January 2017, Pages 216-232, https://doi.org/10.1093/cercor/bhw394

The co-author's name was formerly misspelled as Alexandra Ferrara and this has since been fixed to correctly read as Alexandra Ferrera. The authors apologize for this error. 